\title{
Using AutomationML to Generate Digital Twins of Tooling Machines for the Purpose of Developing Energy Efficient Production Systems
}

\author{
Nicolai BEISHEIM ${ }^{1}$, Markus LINDE, Tobias OTT and Sebastian AMANN \\ Albstadt-Sigmaringen University, Albstadt, Germany
}

\begin{abstract}
The development of energy efficient production systems such as machine tools is a complex process. All specialised departments must work interdisciplinary during the design process in order to achieve an optimal result. In addition to the mechanical aspects, e.g., lightweight construction, the optimization of the Programmable Logic Controller (PLC) programs of tooling machines plays an increasingly important role. By optimizing the programs in terms of energy efficiency, the energy consumption of a machine can be significantly reduced. However, energy consumption depends on many parameters, so the optimization process is complex and a matter of all engineering disciplines working together. By using Digital Twins of tooling machines, simulations can be used to perform many parameter studies for optimizing energy consumption. However, the generation of Digital Twins of production systems is very expensive if they are to represent all relevant features of a production system. Through an IT system of networked software programs and using AutomationML as a special data interface these Digital Twins of machine tools can be generated automatically. The article describes the structure and function of this IT system and how it will be efficient within all involved engineering disciplines.
\end{abstract}

Keywords. AutomationML, Graph-based Design Languages, Digital Twin, Crossdomain Engineering, RAMI 4.0, transdisciplinary development

\section{Introduction}

According to numbers published by Eurostat, the industry is one of the biggest energy consumers in Europe, using $25.8 \%$ of all the Energy, that was consumed over all sectors in 2018 [1]. The European Union wants to decrease the greenhouse gas emissions to at least $55 \%$ compared to 1990 by 2030 as a part of the European Green Deal [2]. To achieve this and to reduce the footprint and the impact on the environment in the future, it will be mandatory, that energy consumption is a key factor within the development process of industrial used machines.

Besides the restrictions that will be made by the governments, another issue is the increasing cost of Energy, especially in Europe, that will make it harder for European

\footnotetext{
${ }^{1}$ Corresponding Author, Mail: beisheim@hs-albsig.de.
} 
companies to compete in the international economy. Companies in Europe will be forced to innovate their production processes in terms of Energy Efficiency.

Optimizing a production system towards Energy Efficiency is a complex task, as there is a big number of factors that impact the result. Therefore, a very close collaboration of all engineering disciplines, that are involved within the development process, e.g., mechanical engineering, electrical engineering, and information technologies, is required.

Simulations as digital twins of technical systems can help to optimize production systems. Many studies can be carried out on digital twins with little effort to determine a balanced relationship between productivity and energy consumption. These studies can also be carried out in parallel with a running production because production of the real system does not have to be interrupted.

However, the model generation of accurate digital twins is complex and there is no unified software platform that represents both the physical characteristics of a plant and its control. So, now data will be passed from one department to another and often needs to be converted, what is time consuming and will result in some type of data loss at any point [3]. The approach presented in this article involves an IT-technical combination of the AutomationML interface format with an automated process of model generation of digital twins using Graph-based Design languages to optimally implement the requirements in the development of energy-efficient production systems. The goal is, to create am IT-System, that integrates all involved engineering disciplines, so an iterative optimization cycle, as shown in Figure 1 can be approached, to optimize a production plant within the very early design phase. To achieve an optimised overall system, it is imperative to create a common data basis for all engineering disciplines to be able to use all the advantages of transdisciplinary cooperation throughout the entire life cycle.

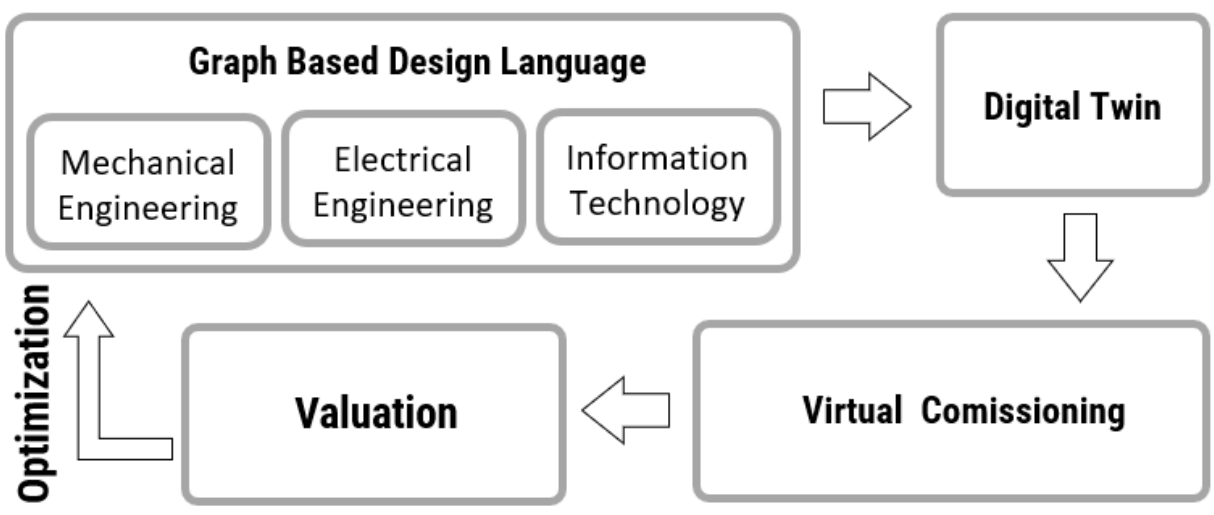

Figure 1. Iterative Cycle of Digital Production Optimization.

\section{Relevant Work}

This chapter will give a brief overview over the fundamental technologies and concepts that were used for this research as well as some hints to some other approaches towards achieving energy efficiency with the help of digital twins. 


\subsection{Production-System Design using Graph-based Design languages}

Graph-based Design languages are used within all kinds of engineering disciplines. They are a useful method to systemize knowledge and therefore achieve interdisciplinary results by systemizing all features of the engineering process. They have three main aspects [4]:

- Vocabulary

Vocabulary is part of abstract knowledge. It describes the available components within the design language and their correlation to each other.

- Rules

The rules form the second part of abstract knowledge, and they are basically the blueprint for the design process. The rules can be influenced by a variety of parameters, which may lead to completely different models.

- Compilers

A compiler is used to create instances of the design described in the vocabulary and rules. The model created by the compiler can then be used as a central model for various other applications.

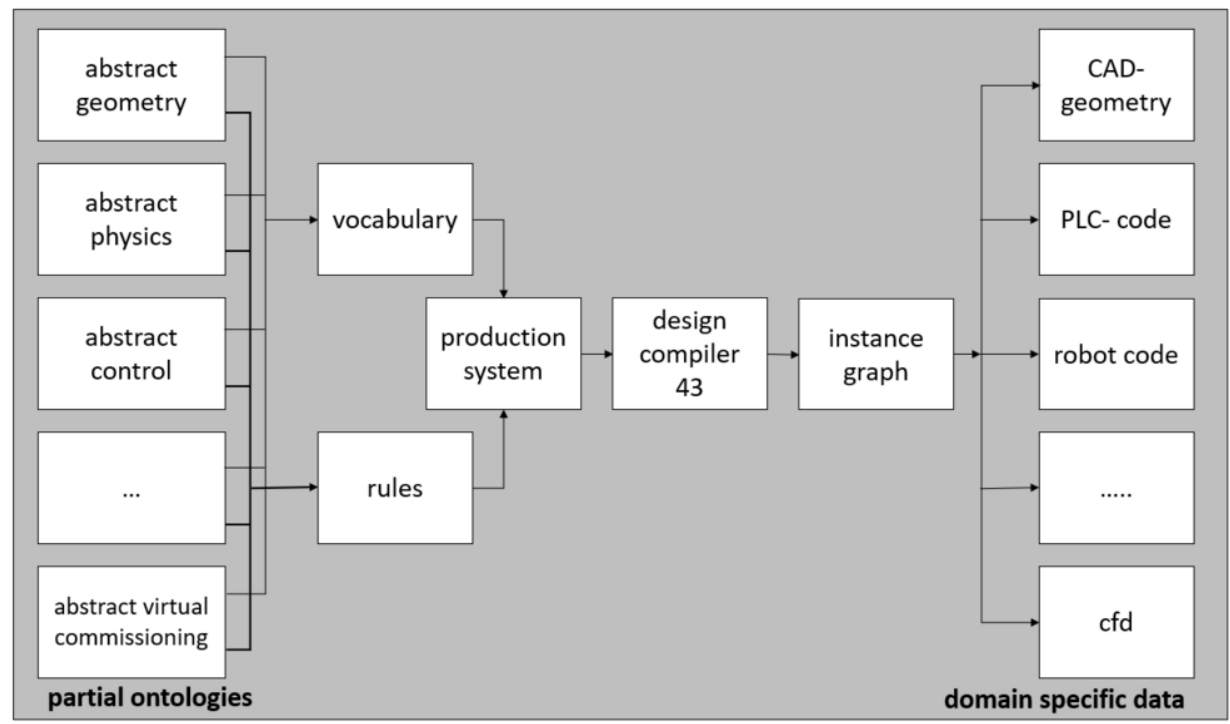

Figure 2. Schematic of the Graph-based Design languages, based on [5].

Figure 2 shows an overview of the structure and functions of Graph-based Design languages. Using these Graph-based Design languages, all functions and properties of a product and the according production system can be generated. For example, a production system that consists of different tooling machines and handling robots can be completely generated, including geometric data, production process flow and the programmable logic controller (PLC) codes, that will be running on the controls of the tooling machines and handling robots. The complete model can then be used along with the programmed controllers for Virtual Commissioning. For this purpose, it is mandatory that all knowledge from all involved engineering disciplines will be systemized using Graph-based Design Languages. 


\subsection{AutomationML}

AutomationML is a data interface in the form of a data container as a file, that allows the departments of different engineering disciplines to easily exchange data. It is an XMLbased format and can store all data, that is relevant for a production system. It can store $3 \mathrm{D}$ CAD geometries as well as PLC codes or robot programs. The exchange is standardized in IEC62713. Because the architecture has already been excessively described in numerous articles e.g., Beisheim et al. [4] [6] [7], only a brief overview is given here.

Figure 3 displays an overview of the structure of the data format of the AutomationML container. The Architecture itself is based on the CAEX-Format, which can be easily extended by linking to other files. Geometric and Kinematic data is stored as COLLADA standard, PLC Programs can be stored as PLCOpen XML. The format can also be extended to store additional data formats [8].

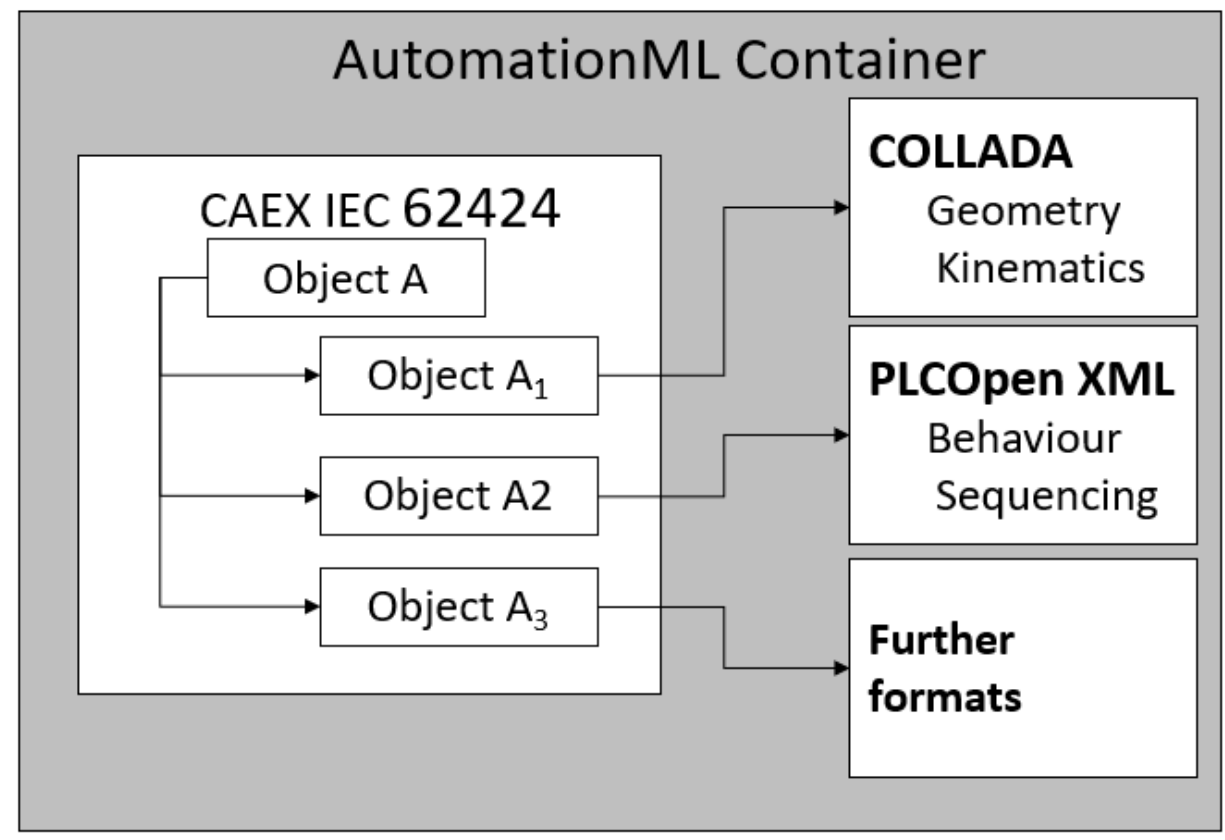

Figure 3. Schematic of the AutomationML container (based on [8]).

\subsection{Attempts to using Digital Twins for Energy Efficiency Optimization}

Using Digital Twins and Virtual Commissioning was a major topic in some recent research, but it is not a very common topic in the industry, as Lechler et al [9] are pointing out. Especially since it is very hard to start developing physical models of production systems, because there are no physical models of the components that are used within the production system available so far. Some attempts that try to increase energy efficiency focus on the process timelines and switching the production systems to standby modes during breaks and downtimes [10]. But to achieve more accurate results, it is mandatory, that the physical behaviour of the production plant is considered as well. 
Damrath et al. [11] describe their approach to energy simulation using Physics Engines, that inherit from the gaming industry. Physics Engines can handle rigid bodies, soft bodies, and fluids. The problem with physics engines is, that most of them calculate the movements and forces based on contact forces. This method is working very well for rigid bodies, but makes the simulation computationally very intense, if fluids or soft bodies need to be considered within the calculation [11].

So, although Physics Engines make Virtual Commissioning more accurate, they still do have limitations, which is why the authors chose a different approach to involve accurate physics in Virtual Commissioning, which will be described in the following sections.

\section{Virtual Comissioning}

In order to be able to determine the energy consumption of a machine using a digital twin, it is imperative to create a very accurate image of the machine using the functions of a Virtual Commissioning model. The model must also have interfaces to the PLC code so that the actual production processes can be examined and optimised.

\subsection{Using Unity3D for Visualisation of a Digital Twin with AutomationML}

Unity3D is a software engine for creating $3 \mathrm{D}$ content and applications. It was originally created as a Game Engine but because of the open software, that Unity provides, it is now used for all kinds of 3D content creation, including Virtual Commissioning purposes. Unity is based on C\# so it can work with many APIs and SDKs for all kinds of use cases. Therefore, Unity3D is an excellent choice for visualisation of Virtual Commissioning Model. A custom developed import extension for AutomationML (AML) files allows the user to load with other software generated files into the simulation environment in Unity3D at runtime. The Collada geometry data contained in the AML file is placed as geometry objects in the scene and each object is assigned its position and functional properties by attributes in the file.

Logic Facets as functions newly developed in Unity3D within the scope of a research project enable the animation of the production process without interfacing with a PLC software. This is very helpful for a first check of the production process or for simple presentation cases. But for an accurate Virtual Commissioning Environment it needs interfaces to commercial software e.g., Beckhoff TwinCAT, so the PLC code can be tested with the Digital Twin of a machine or a robot handling system. For this purpose, interfaces for commercial PLC software were programmed in Unity3D. The interface for Beckhoff TwinCAT is based on the Beckhoff TwinCAT ADS framework, which can be used as an interface with all kinds of $\mathrm{C} \#$ applications.

As shown in Figure 4, the ADS interfaces connect the input and output communication between the TwinCAT PLC Simulation and the Unity3D Simulation Environment. This has created a Digital Twin of a machine or robot that responds to PLC code. Via the ADS interface, the TwinCAT software, for example, receives input signals from events from the Logic Facets in the Unity3D environment, such as the interruption of a light barrier by an object. TwinCAT then triggers programmed actions at the output of the PLC Simulation e.g., that the rotation of a motor shaft should stop. The signal is transferred to Unity3D as input via the ADS connection. The Logic Facet of the motor object then stops the rotation of the motor shaft. In this way, the PLC code can be tested 
and optimised within the Virtual Commissioning Environment to achieve an optimal production process with good energy efficiency.

\section{Virtual Commissioning Environment}

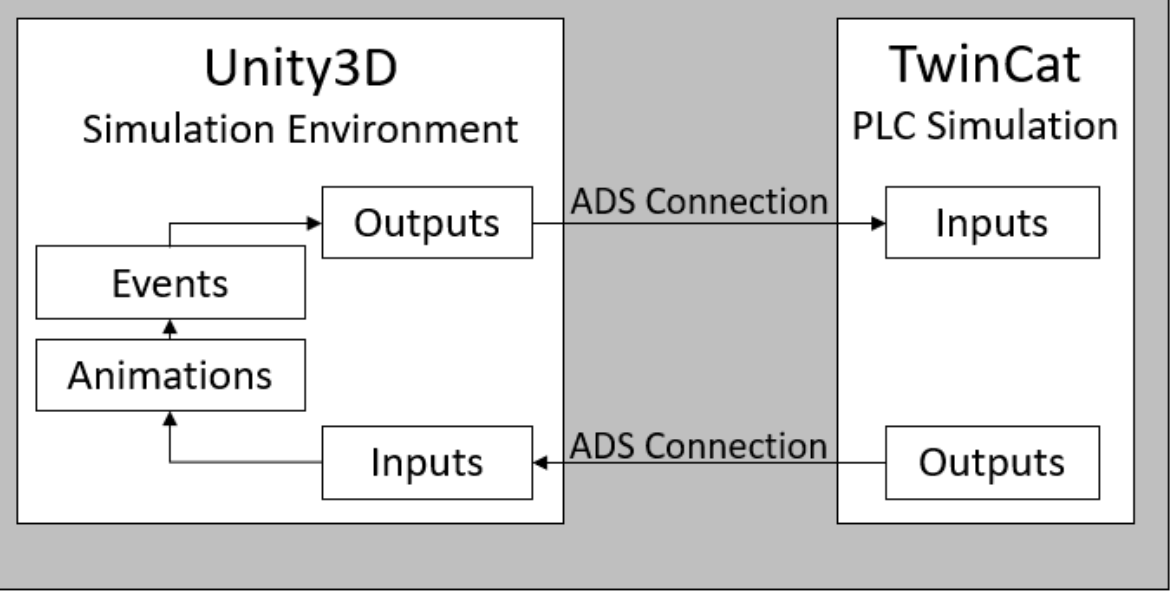

Figure 4. Schematic of the Unity3D - TwinCAT communication.

\subsection{Using ISG Virtuos for physically accurate Virtual Commissioning}

The software Virtuos is a real time simulation software for physically accurate models provided by the Company ISG. Virtuos provides a very large library of mathematical models that can display an accurate behaviour of all kinds of used actors of a production system e.g., hydraulic/pneumatic cylinders, servomotors, gearboxes etc. Using these physically models, it is possible to calculate the energy that is needed for every component, without dealing with the challenges and limitations that come with other Physics Engines.

To be able to use this mathematical model within the Virtual Commissioning Environment, a custom C++ UDP-Interface in Unity3D was developed. The interface is based on the publisher-subscriber principle for communication data. For this communication function, where something can be calculated in Virtuos, a special attribute is added in the AutomationML container. As displayed in Figure 5, the calculations of each process e.g., hydraulic cylinder forward, will be triggered by a write request from the Unity3D Environment. The calculation of the progress is calculated than in Virtuos and the Unity3D Environment will subscribe to the topic, which enables the Simulation Environment to update the actual position of the movement via the UDP interface. The updates will be displayed each frame, what means, that it will update all movements with a framerate of $30 \mathrm{fps}$, so a very fluent visualisation can be achieved. This way, the simulation of the production process can be very detailed and physically accurate.

Virtuos does not support the use of AutomationML files by default so far. However, the program can import a simulation model as an ecf file, but this presents some challenges for automatic modelling. The solution developed in the research project is to create a suitable ecf file outside the program, which then must be imported into Virtuos. 
This file is therefore created by the Graph-based Design language in parallel with the AutomationML file.

\subsection{More Interfaces for more accurate Simulations}

With the interfaces described a solution for a detailed visualisation, accurate physical calculations as well as for the connection to a PLC control software has been achieved. However, to make the Virtual Commissioning environment even more complete, an interface for a commercial robot control software is still needed. The software KUKA.OfficeLite from the company KUKA offers a good solution for virtual robot programming. Therefore, another UDP interface to Unity 3D was developed, which enables communication between the Unity3D Simulation Environment and the Kuka Control Software.

The open architecture of Unity3D allows the creation of a complete virtual commissioning environment with further interfaces to all types of commercial software that will be able to meet many requirements for digital twins of machine tools while working with the AutomationML container. Further interfaces to other types of software for commercial applications can also be added to Unity3D.

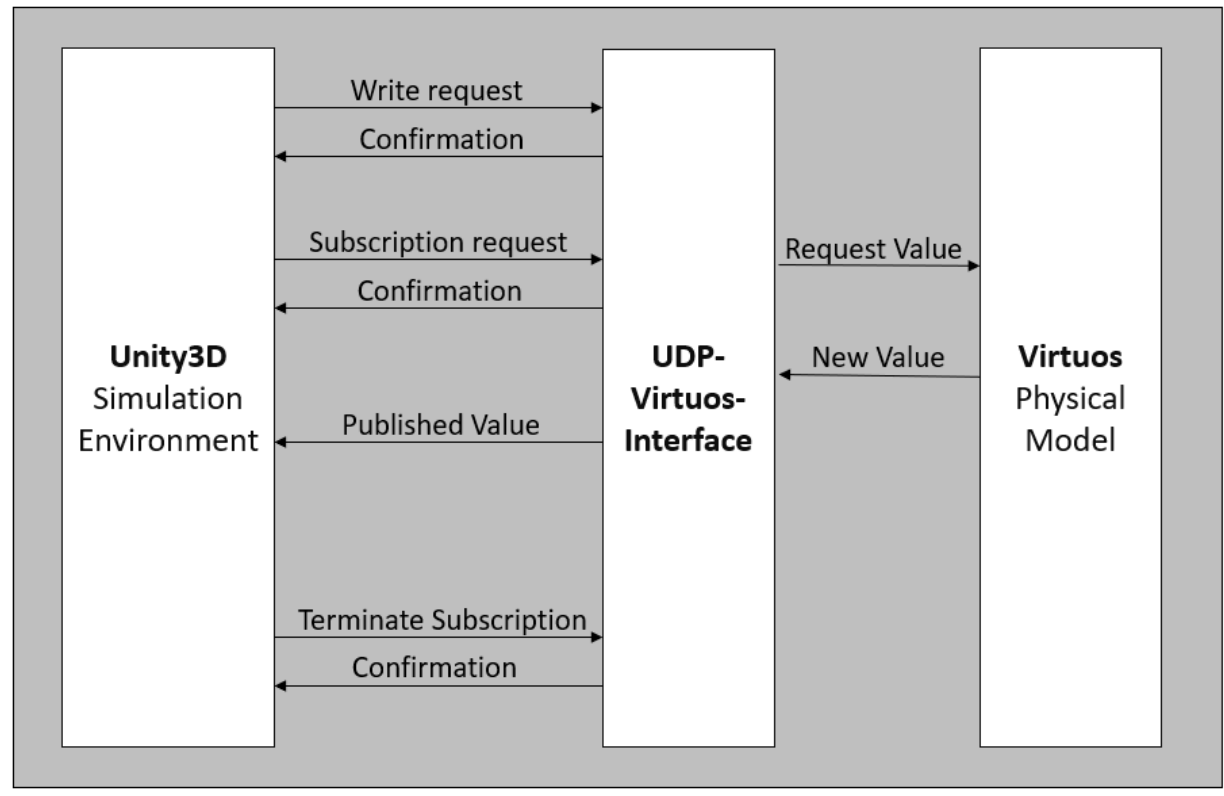

Figure 5. Schematic of the Unity3D - Virtuos communication.

\section{Optimizing the Production Process and Energy Efficiency}

The graph-based design language generates all the data needed for a complete virtual integrated product and production process development. All engineering disciplines can 
work with the same database, which prevents data loss and additional effort for data conversion between different IT tools.

Figure 6 shows the entire IT system with the schematic workflow for optimising a production process and its energy efficiency. The use of a complete IT system makes it possible to carry out many iterations through numerous different variations of the product itself as well as the production system with which it is to be manufactured, even before any form of actual production must take place. During the simulation, pre-defined data is stored. The evaluations of this data are the base for further optimization of the production process and energy efficiency. For this purpose, the parameters are then manually changed in the graph-based design language.

With the help of this IT system, which was developed as part of a research project, products with their production processes can already be developed in the development phase through the application of digital twins and virtual commissioning, which have an optimal relationship between economic, functional and ecological aspects.

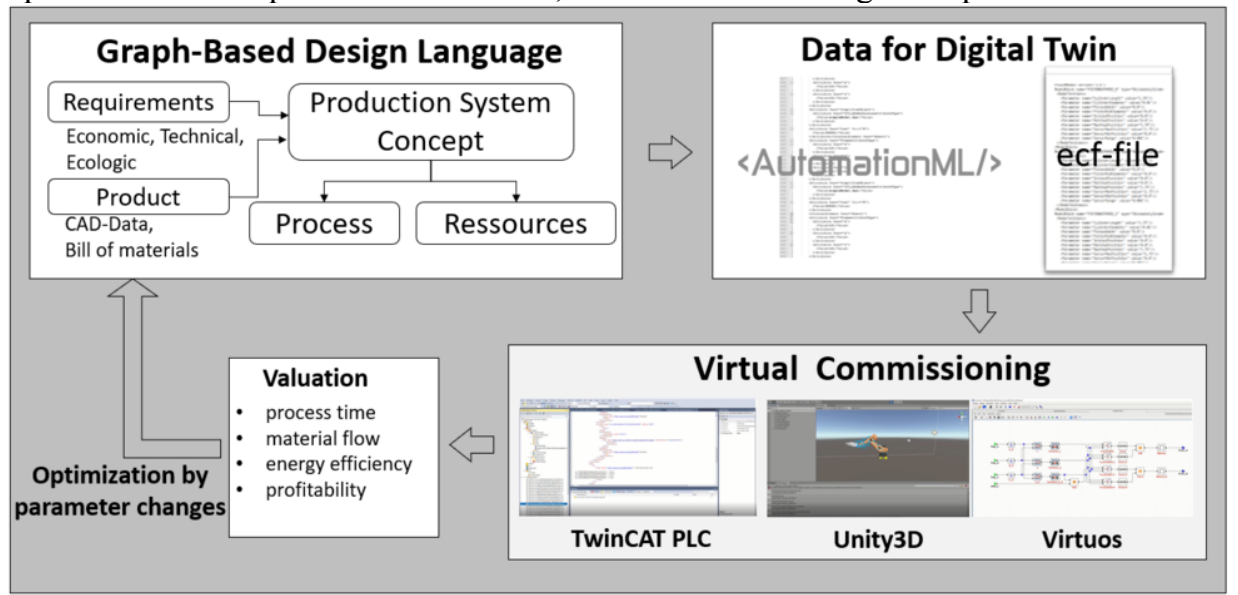

Figure 6. IT system for the optimisation of Product, Production Process and Energy Efficiency.

\section{Application of the IT-System for Virtual Commissioning}

In this section, a small example of the application with a single robot arm will be described, because a complete study of a production system would exeed the volume of this paper. As an example, an industry standard 6 axis robot is used to unload parts from one machine and loading it onto the next machine in the production process.

Given the fact, that there are infinate possibillities on how this small part of the production can be structured, it seems impossible for humans to optimize an entire, complex production plant.

The first task would be to choose the correct robot arm for this purpose, which will primarily be a matter of the required workload, but also of the required workspace, that the robot will need to cover. Choosing the correct robot arm is a simple task that a human can complete without effort. The next step to plan the process of the robot would be to determine the spot where the robot will be placed in the production process. Normally the planning engineer will just choose a place where the robot will be able to reach both points for loading and unloading the workpieces. On Site, there will be a programmer 
who will program the robot arm to load an unload the parts. Often, the robot programmer will just use the point-to-point programming feature of the robot programming software. The trajectory speed will be roughly adjusted to the rest of the process.

The described way to place a robot within a production plant is a simple, straight forward process. However, it does leave room for optimization.

If a production plant is entirely described as a digital twin that was generated by a graph based design language, it is easy to swap out single components. Therefore it is very simple to carry out a big number of parameter studies, where basically every component of the production plant can be swapped or modified, no matter if it is an actual component, PLC code or robot code. This enables an iterative approach, to optimize a production plant in a way, that is needed.

So, the 6 axis robot arm needs to be optimized to use a minimal amount of energy, without causing a bottleneck in production capacity. The robot arm is physically described in an .ecf file, that is loaded into the Virtuos Software. It consists of frequency converters, synchomotors, gear drives and the axes of the robot. The code of the robot is saved in the AML file and imported into the robot control software (e.g. KukaOffice Light). The geometries are then loaded into the visualization environment in Unity3D that also handles all events and acts as the center of the virtual comissioning IT system. The trajectories are given by the robot program, that connects to Unity3D. There the trajectories will be shown according to the calculations in Virtuos. Given the required speed and acceleration of the robot arm, determined by the robot code, as well as the robot axes and the weight at the Tool Center Point, the needed energy can be calculated by Virtuos. An iterative process, where the position and the robot, as well as the trajectories, speeds and accellerations can be adjusted, enable the development team to find the optimal parameters by testing a large number of different combinations.

The robot speed can therefore be adjusted precisely to the rest of the production process. So unneccesarily high accelerations and speeds can be avoided.

To confirm the results of the digital optimization, an existing robot arm in the laboratory of the university was equipped with sensors to collect data of accelerations in the tool center point and also measure current on all frequency converters simultaneously. The measurements show, that the robot did use less energy when it used the optimized movement commands, because they used slower speeds, so the robot never has any pause times, but just fulfills the requirements to move the parts, so the rest of the plant also does not suffer from a bottleneck that is created by slow trajectory speeds.

\section{Conclusion and Further Research}

The mapping of machines as Digital Twins and used in Virtual Commissioning Environment have a great potential to optimise products and production processes. Such an IT system consisting of a Graph-based Design language, AutomationML interface and visualisation in Unity3D with interfaces to PLC, Physics Engine and robot controllers enables closer cooperation between the various engineering disciplines.

The automatic generation of Digital Twins by Graph-based Design languages, as described in detail in Kiesel et al. [6] and Beisheim et al. [7], enables the rapid development of different variants of simulation models of the products and processes for parameter studies e.g., regarding energy efficiency. This automatic generation also reduces the effort of using Digital Twins for optimization in the development phase of a new product and its production processes. The usage of Graph-based design language as 
an instrument to systemize (capturing, storing and providing) knowledge off all product engineering disciplines makes it easier for the departments to collaborate interdisciplinary.

The use of the Virtuos software integrated into the IT system enables the calculation of the physical relationships of the simulation objects. However, it also increases the effort to create the models. A future improved availability of simulation models of components such as motors, pneumatic cylinders, etc., which are made available online by the component manufacturers, will significantly reduce the effort for model creation. In further research projects, the IT system is being validated using data from machines and processes from the industry. And through further functional developments of the overall system, the degree of automation is constantly being increased.

\section{Acknowledgement}

The German research project 'Digitaler Produktlebenszyklus (DiP)' (information: http://dip.reutlingen-university.de) is supported by a grant from the European Regional Development Fund and the Ministry of Science, Research and the Arts of BadenWürttemberg, Germany (information: http://www.rwb-efre.baden-wuerttemberg.de).

\section{References}

[1] European Commission/Eurostat, 2019, Final energy consumption by sector, EU-27, 2018 (\% of total, based on tonnes of oil equivalent), Accessed: 15.02.2020. [Online]. Available:

https://ec.europa.eu/eurostat/statistics-

explained/index.php?title=File:Final_energy_consumption_by_sector,_EU27,_2018_(\%25_of_total, based_on_tonnes_of_oil_equivalent).png

[2] European Commission, 2020, 2030 climate \& energy framework, Accessed: 15.02.2020. [Online]. Available: https://ec.europa.eu/clima/policies/strategies/2030

[3] N. Beisheim, T. Ott, S. Amann, Einsatz von AutomationML zur automatischen Generierung von Digitalen Zwillingen von Werkzeugmaschinen zur Entwicklung energieeffizienter Produktionssysteme. 26. Interdisziplinäre Wissenschaftliche Konferenz, Mittweida, April 14th-15th, 2021.

[4] N. Beisheim, M. Kiesel, S. Rudolph, Digital manufacturing and virtual commissioning of Intelligent Factories and Industry 4.0 systems using graph-based design languages, Advances in Transdisciplinary Engineering, 2018, Vol. 7, pp. 93-102

[5] S. Rudolph, On the problem of multi-disciplinary system design - and a solution approach using graphbased design languages, 1st ACCM Workshop on Mechantronic Design, Linz, November 30, 2012.

[6] M. Kiesel, P. Klimant, N. Beisheim, S. Rudolph, M. Putz, Using Graph-based Design Languages to Enhance the Creation of Virtual Commissioning Models, Procedia CIRP, Vol. 60, 2017, pp. 279-283.

[7] N. Beisheim, M. Kiesel, M. Linde, T. Ott, Using AutomationML and graph-based design languages for automatic generation of digital twins of cyber-physical systems, Advances in Transdisciplinary Engineering, 2020, Vol. 12, pp. 135-142.

[8] AutomationML e. V., 2016, Standardized data exchange in the engineering process of production systems. Accessed: 15.01.2020. [Online]. Available: https://www.automationml.org/o.red/uploads/dateien/1544706233-automationml.pdf

[9] T. Lechler, E. Fischer, M. Metzner, A. Mayr, J. Franke, Virtual Commissioning - Scientific review and exploratory use cases in advanced production systems. $52^{\text {nd }}$ CIRP Conference on Manufacturing Systems, Ljubljana, Slovenia, June 12-14, 2019.

[10] S. Mechs, S. Lamparter, J.P. Müller, J. Peschke, Networked Priced Timed Automata for Energyefficient Factory Automation, Proceedings of the American Control Conference, June 2012.

[11] F. Damrath, A. Strahilov, T. Bär, M. Vielhaber, Establishing Energy Efficiency as Criterion for Virtual Commissioning of Automated Assembly Systems, Procedia CIRP, Vol. 23, 2014, pp. 137-142. 